\title{
Geographic variations of human papilloma virus infection and their possible impact on the effectiveness of the vaccination programme
}

\author{
IOANNIS N. MAMMAS ${ }^{1}$, DIMITRA VAGELI ${ }^{2}$ and DEMETRIOS A. SPANDIDOS ${ }^{1}$ \\ ${ }^{1}$ Laboratory of Virology, Faculty of Medicine, University of Crete, Heraklion, Crete; \\ ${ }^{2}$ Department of Pathology, School of Medicine, University of Thessaly, Larissa, Greece
}

Received April 2, 2008; Accepted May 5, 2008

\begin{abstract}
Greece is one of the first countries of the European Union to introduce a human papilloma virus (HPV) vaccine in its national vaccination programme. Geographical variations in the prevalence of the different HPV types have been demonstrated. The aim of this preliminary case control study was to investigate HPV infection in women with low- and high-grade squamous intraepithelial lesions (SILs) from two different geographical areas of Greece; Central Greece and Crete. Seventy-five cervical specimens were collected from women with SILs from Crete and Central Greece. HPV detection and typing were performed by using polymerase chain reaction (PCR) techniques. HPV-18 was detected more frequently in Crete than in the Central Greece samples (29.7 vs $13.1 \%$ ). HPV-16 was predominant in Central Greece (34.2\%), while in Crete it was detected in $23 \%$ of the studied women. Non-16/-18 HPV types were detected in $45.9 \%$ of the women from Crete and $52.6 \%$ of the women from Central Greece. No relationship was observed between the geographical distribution of HPV and the presence of K-ras or B-raf point mutations in either group. Our pre-vaccination data indicate a high prevalence of HPV-18 in Crete. A trend for difference was observed in the rates of non-HPV-16/-18 women between the two areas. A large epidemiological study is required to investigate the prevalence of the different HPV types to further investigate the effectiveness of HPV vaccination in the Greek population.
\end{abstract}

\section{Introduction}

Human papilloma viruses (HPV) are small double-stranded DNA viruses that comprise a remarkably heterogeneous

Correspondence to: Professor D.A. Spandidos, Laboratory of Virology, Faculty of Medicine, University of Crete, Heraklion, Crete, Greece

E-mail: spandidos@spandidos.gr

Key words: human papilloma virus, vaccination programme, geographic variations, Greece family of more than 130 types $(1,2)$. Different HPV types can cause a wide range of infections in adults and children, including common warts, genital warts, recurrent respiratory papillomatosis, low-grade and high-grade squamous intraepithelial lesions and cervical cancer. 'High-risk' HPV types have been implicated in the development of intraepithelial lesions (SILs) and its progression to cervical cancer $(2,3)$. Fifteen HPV types have been classified as 'high risk', HPV$16,-18,-31,-33,-35,-39,-45,-51,-52,-56,-58,-59,-68,-73$ and $-82(4,5)$.

It has been demonstrated that the distribution of different HPV types in women with cervical neoplasia varies according to region (6-8). These geographic variations can play an important role in the effectiveness of an HPV vaccination and must be taken into consideration when designing policies against HPV. Current and proposed HPV vaccine candidates are HPV type-specific conferring only type-specific immunity against 'high-risk' types HPV-16 and -18 (9-11). HPV-16 and -18 are the most common HPV types and are responsible for $\sim 70 \%$ of all cervical cancer cases $(5,6)$. Vaccination against HPV-16 and -18 potentially prevents more than two thirds of cervical cancer cases worldwide. However, the impact of an HPV vaccination in different geographical regions will be related to the prevalence of HPV-16 and -18 in the different populations (6).

Interestingly, among women with low- and high-grade SILs, the prevalence of HPV-16/-18 is lower compared to cervical cancer; however, it follows similar patterns in different regions $(6,7)$. The aim of this preliminary case control study was to investigate HPV infection in women with low-grade and high-grade SILs from two different geographical areas of Greece, the island of Crete and Central Greece. We also examined the presence of K-ras codon 12 and B-raf exon 15 point mutations and their possible relationship to the geographical distribution of HPV. Greece is one of the first European Union countries to introduce an HPV vaccine in its national vaccine schedule after the approval of the quadrivalent vaccine by the European Union in 2006. Prevaccination data concerning the prevalence of HPV genital infection in women will enable the investigation of the effectiveness of an HPV vaccination and to prevent HPVinduced carcinogenesis. 


\section{Materials and methods}

Our study included cervical specimens that were obtained from women with low-grade or high-grade cervical intraepithelial lesions (SILs), who were treated at the University Hospital of Heraklion on the island of Crete and the University Hospital of Larissa in Central Greece during the period 2003 to 2005. The histological analysis of the material was performed at the Departments of Pathology of the University Hospitals of Heraklion and Larissa, Greece. Clinicopathological data (age, origin, residence and medical history) were available for all patients included in the study. Tissue specimens obtained during colposcopy were divided into two parts. One portion was fixed in buffered formalin $(\mathrm{pH}$ 7.4) for routine histopathological assessment, while the other portion was paraffin-embedded for DNA extraction. DNA was extracted as previously described (12) and stored at $-20^{\circ} \mathrm{C}$.

For the detection of the HPV the general primers GP5 ${ }^{+}$ and $\mathrm{GP}^{+}$were used. The extracted DNA $(1 \mu \mathrm{l})$ of each sample was amplified in a total volume of $30 \mu \mathrm{l}$ containing $5 \mu \mathrm{M}$ of 10X PCR reaction buffer $(200 \mathrm{mM}$ Tris- $\mathrm{HCl}, \mathrm{pH} 8.4$ and $500 \mathrm{mM} \mathrm{KCl}), 1.5 \mathrm{mM} \mathrm{MgCl}_{2}, 200 \mu \mathrm{M}$ of each dNTP, $0.5 \mu \mathrm{M}$ of each primer and $0.6 \mathrm{U}$ of recombinant Taq DNA polymerase (Invitrogen Ltd., UK).

HPV typing was performed by using separately specific pairs of primers for virus type HPV-16 and HPV-18. The extracted DNA $(1 \mu 1)$ of each sample was amplified in a total volume of $20 \mu \mathrm{l}$ containing $1 \mathrm{X}$ PCR reaction buffer, $1.5 \mathrm{mM}$ $\mathrm{MgCl}_{2}, 200 \mu \mathrm{M}$ of each dNTP, $0.5 \mu \mathrm{M}$ of each primer (sense and antisense) and 0.6 U Platinum Taq DNA polymerase (Life Technologies Ltd., UK). The amplification conditions, the general primers for HPV detection, the specific primers used for HPV detection and typing have been previously described $(13,14)$. PCR products for HPV were analysed on $2 \%$ agarose gel and photographed on a UV light transilluminator. PCR assay was carried out in a PTC-200 programmable thermal controller (MJ Research Inc., USA). All PCR reactions included appropriate negative controls. DNA extracted from HeLa cells and plasmid DNA of HPV-16 and HPV-18 were used as positive controls. The detection of exon $15 \mathrm{~B}-r a f$ and codon $12 \mathrm{~K}$-ras point mutations was performed using polymerase chain reaction (PCR) and restriction fragment length polymorphism (RFLP) analysis as previously described (15).

Statistical analyses were performed using SPSS software (SPSS, version 11.5). The Pearson $\chi^{2}$ test was performed in order to compare the two different group populations according to age, origin, residence, histology, point K-ras and B-raf mutations and HPV status. The limit of statistical significance was set at $\mathrm{p}<0.05$.

\section{Results}

Our study included 75 Greek women with squamous epithelial neoplasia (SIL). The clinicopathological characteristics of our sample are presented in Table I. In the population group from Crete, 23 women had low-grade SILs and 14 had highgrade SILs. Of the women from Central Greece, 23 had lowgrade SILs and 15 had high-grade SILs. The mean age of the population groups from Crete and Central Greece was 42
Table I. Clinicopathological characteristics of the sample of our study $(\mathrm{n}=75)$.

\begin{tabular}{lccc}
\hline Characteristic & Crete & Central Greece & p-value \\
\hline $\begin{array}{l}\text { Specimens } \\
\text { Cervical smears }\end{array}$ & $37(100 \%)$ & $38(100 \%)$ & \\
Histology & & & \\
LSIL & $23(62.2 \%)$ & $23(60.5 \%)$ & \\
HSIL & $14(37.8 \%)$ & $15(39.5 \%)$ & NS \\
Age & & & \\
$<35$ years & $18(48.6 \%)$ & $17(44.7 \%)$ & \\
$\geq 35$ years & $19(51.4 \%)$ & $21(55.3 \%)$ & NS \\
$\begin{array}{l}\text { Ethnicity } \\
\text { Caucasian }\end{array}$ & $37(100 \%)$ & $38(100 \%)$ & \\
Other & $0(0 \%)$ & $0(0 \%)$ & NS \\
Residence & & & \\
Urban & & & \\
Rural & $27(73 \%)$ & $26(68.4 \%)$ & NS \\
K-ras status & $10(27 \%)$ & $12(31.6 \%)$ & NS \\
Codon 12 \\
mutation $(+)$
\end{tabular}

Pearson $\chi^{2}$ test; NS, not significant ( $p>0.05$ ). LSIL, low-grade squamous intraepithelial lesions; HSIL, high-grade squamous intraepithelial lesions.

(range 28-58) years and 44 (range 31-61) years, respectively. No differences were observed between samples from Crete and Central Greece according to age, ethnicity or residence (Pearson $\chi^{2}$ test, $\mathrm{p}>0.05$ ). Codon 12 point mutations in $\mathrm{K}$-ras were detected in 4 out of 37 women from Crete and 5 out of 38 from Central Greece. All K-ras mutations were detected in women with high-grade SILs. No B-raf exon 15 point mutation was detected in either population. There was no statistically significant difference in the presence of $\mathrm{K}$-ras or B-raf mutation between samples from Crete and Central Greece (Table I).

The results of the HPV detection and typing of our study samples are presented in Table II. All women included in our study were HPV-positive $(75 / 75,100 \%)$. HPV-18 was detected in 11 women $(29.7 \%)$ from Crete and in 5 women $(13.1 \%)$ from Central Greece. HPV-16 was predominant in Central Greece (34.2\%), while in Crete it was detected in 9 women (27\%). Non-16/-18 HPV types were detected in 17 $(45.9 \%)$ women from Crete and 20 (52.6\%) women from Central Greece; however, this difference was not statistically significant (Pearson $\chi^{2}$ test, $\mathrm{p}>0.05$ ).

In the total sample, HPV-16 was detected more frequently than HPV-18 (30.7 vs 21.3\%). HPV-16 was detected in 10 $(21.7 \%)$ women with low-grade SILs and in $44.8 \%$ of women 
Table II. HPV detection and typing in women with squamous intraepithelial neoplasia in two geographical areas of Greece: Central Greece vs Crete.

\begin{tabular}{|c|c|c|c|c|}
\hline \multirow[t]{2}{*}{ Sample group } & \multicolumn{4}{|c|}{ HPV status } \\
\hline & HPV (+) & HPV-16 (+) & HPV-18 (+) & Non-HPV-16/-18 (+) \\
\hline \multicolumn{5}{|l|}{ Crete } \\
\hline LSIL & 23 & 5 & 5 & 13 \\
\hline HSIL & 14 & 5 & 6 & 4 \\
\hline Total & $37(100 \%)$ & $10(27.0 \%)$ & $11(29.7 \%)$ & $17(45.9 \%)$ \\
\hline \multicolumn{5}{|l|}{ Central Greece } \\
\hline LSIL & 23 & 5 & 2 & 16 \\
\hline HSIL & 15 & 8 & 3 & 4 \\
\hline Total & $38(100 \%)$ & $13(34.2 \%)$ & $5(13.1 \%)$ & $20(52.6 \%)$ \\
\hline \multicolumn{5}{|l|}{ Total } \\
\hline LSIL & 46 & 10 & 7 & 29 \\
\hline HSIL & 29 & 13 & 9 & 8 \\
\hline Total $(\%)$ & $75(100 \%)$ & $23(30.7 \%)$ & $16(21.3 \%)$ & $37(49.3 \%)$ \\
\hline
\end{tabular}

LSIL, low-grade squamous intraepithelial lesions; HSIL, high-grade squamous intraepithelial lesions.

with high-grade SILs. The respective rates for HPV-18 among patients with low- and high-grade SILs were 15.2 and $31.3 \%$. Co-infection with HPV-16 and -18 was detected in one woman with high-grade SIL from Crete. In the total sample, the ratio HPV-16/-18 to non-HPV-16/-18 was 1.03 (38/37), the ratio among women with low-grade SILs was 0.55 (16/29), and the ratio among women with high-grade SILs was 2.63 (21/8). The respective ratios among women from Crete were 1.17 (20/17), 0.77 (10/13) with low-grade SILs, and 2.5 (10/4) with high-grade SILs, and among women from Central Greece 0.9 $(18 / 20), 0.44$ (7/16) with low-grade SILs, and $2.75(11 / 4)$ with high-grade SILs.

\section{Discussion}

The human papilloma virus (HPV) has a global distribution (6). The prevalence of the different types of HPV worldwide has implications for the effectiveness of HPV vaccinations against HPV-induced carcinogenesis. This is the first report in the literature to document differences in the geographical distribution of HPVs in Greece. It has been proposed that a worldwide vaccine against HPV-16/-18 may prevent a larger proportion of cervical carcinoma cases in Europe, North America and Australia than in Africa, South America and Asia $(6,8)$.

HPV-16 represents the most commonly identified HPV type in low- and high-grade SILs as well as cervical cancer worldwide (6-8). A recent meta-analysis of low-grade SILs by Clifford et al showed that the prevalence of HPV-16 ranges from $9 \%$ in Africa to $21 \%$ in Asia, while in Europe the prevalence is $19 \%$ (7). A similar meta-analysis of highgrade SILs found a prevalence of HPV-16 of $32 \%$ in Africa, $37 \%$ in South America, $46 \%$ in North America and 53\% in Europe (8). The predominance of HPV-16 has also been demonstrated in cases with squamous cervical cancer, and the prevalence of HPV-16 varies consistently being highest in Europe and lowest in Africa (6). Notably, in our sample from Crete, HPV-16 was detected in $21.7 \%$ in low-grade SILs and $35.7 \%$ in high-grade SILs, while among women from Central Greece the respective prevelance was $21.7 \%$ in lowgrade SILs and $53.3 \%$ in high-grade SILs. It seems that HPV-16, which dominates in mainland Europe and worldwide, is detected less frequently in women from Crete.

Among low-grade SILs, the prevalence of HPV-18 has previously been estimated as $5.3 \%$ in Africa, $7.1 \%$ in Asia, 9.2\% in North America, 3.6\% in South/Central America and $5.2 \%$ in Europe (7). Among high-grade SILs, the respective prevalence ranges from $6.5 \%$ in Europe to $10 \%$ in North America, while the pattern is consistent among SCC $(6,8)$. In our sample, HPV-18 was detected in $21.7 \%$ of low-grade SILs and $42.9 \%$ of high-grade SILs. The frequency of HPV18 in the sample from Crete was higher compared to the frequencies that have been reported by others in Europe. Notably, the frequency of HPV-18 found in women with SIL from Crete is one of the highest that has been reported in the literature $(6,8)$. This finding is in agreement with studies in Greece that have demonstrated a high frequency of HPV-18 genital infection in the Greek population as summarised in Table III. Further research is required to study the possible causes of HPV-18 dominance in Crete.

The distribution of HPV types in different geographical regions may be related to host immunologic and genetic factors (16). Crete, an island at the southeast edge of Europe, close to Africa, represents a remote population target with unique geographical characteristics. Knowledge on the distribution of different HPV types in a region is essential for designing and implementing an effective HPV vaccination programme. Currently, the only 'high-risk' HPV types that are covered by the different current virus-like particle vaccines are HPV-16 and -18 $(9,10)$. The geographical distribution of 


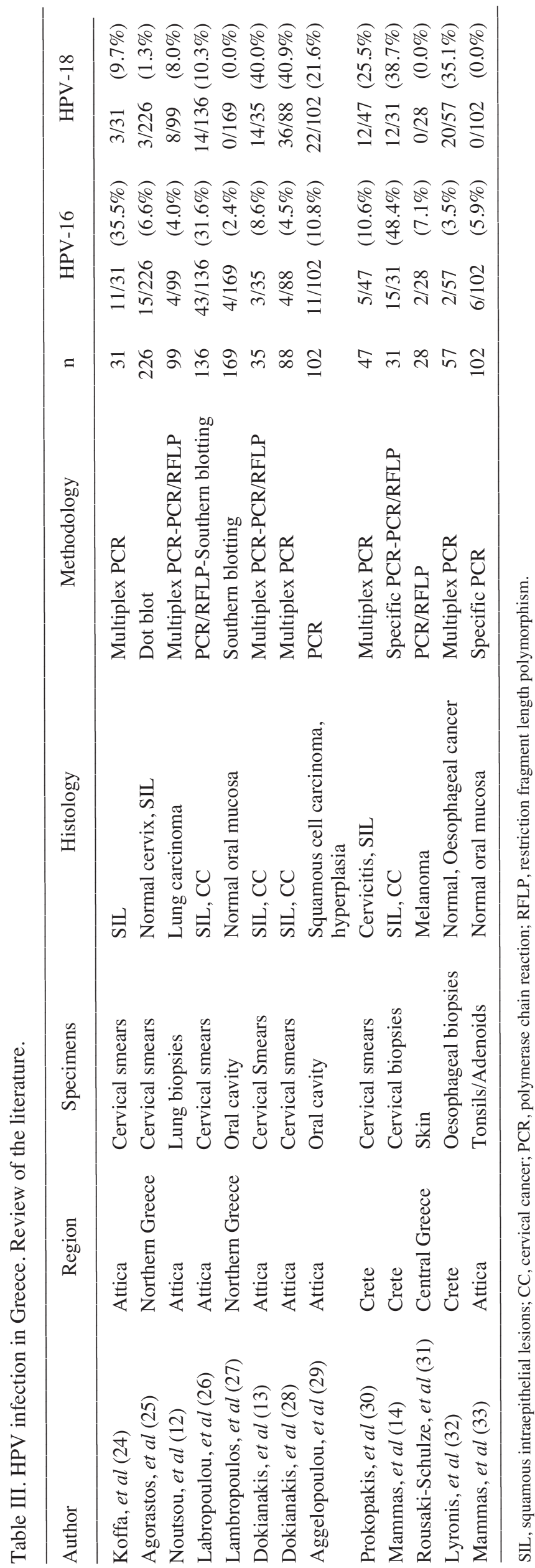

different HPV types suggests that the effect of vaccination against HPV will vary depending on the region.

No correlation was found in HPV distribution and K-Ras/ B-Raf mutational activation. The Ras-Raf signaling pathway is a key membrane-to-nucleus signaling system that regulates fundamental cellular functions in response to extracellular signals by activating a number of effector proteins $(17,18)$. Mutational activation of the Ras-Raf signaling pathway at different levels has been implicated in the initiation and progression of several types of human cancer $(19,20)$. K-ras mutations occur in the early stages of cervical neoplasia and represent the most frequent ras point mutations in women with SIL (21). B-raf mutations have recently been found in several types of cancer and have been proposed as a common mechanism of Ras-Raf signaling pathway activation which leads to cellular transformation (22). However, the absence of B-Raf mutations in our population indicates that B-Raf mutational activation does not seem to be involved in the pathogenesis of SIL.

Although HPV-16 and -18 are the dominant HPV types detected in women with cervical cancer and its precursors worldwide, in several geographical areas, other HPV types have been detected more frequently than HPV-16 and -18 $(6,7)$. Among low-grade SILs, other 'high-risk' HPV types, such as $-31,-51,-52,-56$ and -58 were detected more frequently than HPV-18 (7). In Asia, a high prevalence of HPV-58 and in Europe HPV-31 has been demonstrated in low-grade and high-grade SILs (6-8). Among women with SILs, the frequency of non-16/-18 HPV types ranges from 34 to $68 \%$. High prevalence of non-16/-18 HPV types are of great importance since these types are not covered by the existing vaccines. In our study, the ratio HPV-16/-18 to nonHPV-16/-18 in Central Greece [0.9 (18/20)] was lower compared to the ratio of $1.17(20 / 17)$ in Crete.

Both current and future HPV vaccine candidates are HPV type-specific conferring only type-specific immunity (9-11). Data on cross-protection of the current HPV L1 virus-like particle vaccines against other HPV types are still limited. It has been shown that prophylactic quadrivalent human papilloma-virus (types $-6,-11,-16$, and -18)-like particle vaccine results in a neutralizing antibody response which is HPV type specific (9). Recently, Harper et al reported that the protection conferred by the bivalent virus-like particle vaccine against HPV-16 and -18 was extended to 'high-risk' types HPV-45 and -31 (10). Taxonomically, the DNA genome of different types of HPV differs by at least $10 \%$ of the nucleotide sequence of the three open reading frames E6, E7 and L1 from that of any other known type (23). Long-term follow-up studies will clarify the role of HPV vaccination against non-16/-18 HPV types.

The differences in the geographical distribution of HPV types in Greece remain to be clarified. In the future, it is crucial to examine a larger number of clinical samples and to detect the prevalence of all fifteen 'high-risk' HPV types in the Greek population. These findings will provide prevaccination data of the population enabling the evaluation of the efficacy of the vaccination programme and to promote policies against HPV-induced carcinogenesis in Greece. Moreover, possible geographical variations in specific areas demonstrate the necessity for the development of polyvalent 
heterogeneous vaccines in the future which will include types that are more prevalent in specific regions.

\section{References}

1. Sanclemente G and Gill DK: Human papillomavirus molecular biology and pathogenesis. J Eur Acad Dermatol Venereol 16: 231-240, 2002

2. zur Hausen H: Papillomaviruses and cancer: from basic studies to clinical application. Nat Rev Cancer 2: 342-350, 2002.

3. Peto J, Gilham C, Deacon J, et al: Cervical HPV infection and neoplasia in a large population-based prospective study: the Manchester cohort. Br J Cancer 91: 942-953, 2004.

4. Munoz N, Bosch FX, de Sanjose S, et al: Epidemiologic classification of human papillomavirus types associated with cervical cancer. N Engl J Med 348: 518-527, 2003.

5. Munoz N, Bosch FX, Castellsague X, et al: Against which human papillomavirus types shall we vaccinate and screen? The international perspective. Int J Cancer 111: 278-285, 2004.

6. Clifford GM, Smith JS, Plummer M, Munoz N and Franceschi S: Human papillomavirus types in invasive cervical cancer worldwide: a meta-analysis. Br J Cancer 88: 63-73, 2003.

7. Clifford GM, Rana RK, Franceschi S, Smith J, Gough G and Pimenta JM: Human papillomavirus genotype distribution in low-grade cervical lesions: comparison by geographic region and with cervical cancer. Cancer Epidemiol Biomarkers Prev 14: 1157-1164, 2005.

8. Clifford GM, Smith JS, Aguardo T and Franceschi S: Comparison of HPV type distribution in high-grade cervical lesions and cervical cancer: a meta-analysis. Br J Cancer 89: 101-105, 2003.

9. Villa LL, Coste RL, Petta CA, et al: Prophylactic quadrivalent human papillomavirus (types 6,11,16, and 18) L1 virus-like particle vaccine in young women: a randomised double-blind placebo-controlled multicentre phase II efficacy trial. Lancet Oncol 6: 271-278, 2005 .

10. Harper DM, Franco EL, Wheeler CM, et al: Sustained efficacy up to 4.5 years of a bivalent L1 virus-like particle vaccine against human papillomavirus types 16 and 18: follow-up from a randomised control trial. Lancet 367: 1247-1255, 2006.

11. Galloway DA: Papillomavirus vaccines in clinical trials. Lancet Infect Dis 3: 469-475, 2003.

12. Noutsou A, Koffa M, Ergazaki M, Siafakas N and Spandidos DA: Detection of human papilloma virus (HPV) and K-ras mutations in human lung carcinomas. Int J Oncol 8: 1089-1093, 1996.

13. Dokianakis DN, Sourvinos G, Sakkas S, Athanasiadou E and Spandidos D: Detection of HPV and ras gene mutations in cervical smears from female genital lesions. Oncol Rep 5: 1195-1198, 1998.

14. Mammas IN, Zafiropoulos A, Sifakis S, Sourvinos G and Spandidos DA: Human Papilloma Virus (HPV) typing in relation to ras oncogene mRNA expression in HPV-associated human squamous cervical neoplasia. Int J Biol Markers 20: 257-263, 2006.

15. Symvoulakis EK, Zavarinos A, Panutsopoulos D, Zoras O, Papalambros E, Sigala F and Spandidos DA: Highly conserved sequence of exon $15 \mathrm{BRAF}$ gene and KRAS codon 12 mutation among Greek patients with colorectal cancer. Int J Biol Markers 22: 12-18, 2007.
16. Hildesheim A and Wang SS: Host and viral genetics and risk of cervical cancer: a review. Virus Res 89: 229-240, 2002.

17. Chong H, Vikis HG and Guan KL: Mechanisms of regulating the Raf kinase family. Cell Signal 15: 463-469, 2003

18. Macaluso M, Russo G, Cinti C, Bazan V, Gebbia N and Russo A Ras family genes: an interesting link between cell cycle and cancer. J Cell Physiol 192: 125-130, 2002.

19. Davies H, Bignell GR, Cox C, et al: Mutations of the BRAF gene in human cancer. Nature 417: 949-954, 2002.

20. Kiaris H and Spandidos DA: Mutations of ras genes in human tumours. Int J Oncol 7: 413-421, 1995.

21. Mammas IN, Zafiropoulos A and Spandidos DA: Involvement of ras genes in female genital tract cancer (Review). Int J Oncol 26: 1241-1255, 2005.

22. Salvesen HB, Kumar R, Stefansson I, et al: Low frequency of BRAF and CDKN2A mutations in endometrial cancer. Int $\mathbf{J}$ Cancer 115: 930-934, 2005.

23. Chan SY, Delius H, Halpern L and Bernard HU: Analysis of genomic sequencies of 95 papillomavirus types: uniting typing, phylogeny, and taxonomy. J Virol 69: 3074-3083, 1995.

24. Koffa M, Koumantakis E, Ergazki M, Malamou-Mitsi V and Spandidos DA: Detection of ras mutations and HPV in lesions of the human female reproductive tract. Int J Oncol 5: 189-195, 1994.

25. Agorastos T, Bontis J, Lambropoulos A, et al: Epidemiology of human papillomavirus infection in Greek asymptomatic women. Eur J Cancer Prev 4: 159-167, 1995.

26. Labropoulou V, Diakomanolis E, Dailianas S, Kalpaktsoglou K, Balamotis A and Mavromara P: Type-specific prevalence of genital human papillomaviruses in benign, premalignant and malignant biopsies in patients from Greece. Sex Transm Dis 24: 467-474, 1997.

27. Lambropoulos A, Dimitrakopoulos J, Frangoulides, et al: Incidence of human papillomavirus $6,11,16,18$ and 33 in normal oral mucosa of a Greek population. Eur J Oral Sci 105: 294-297, 1997.

28. Dokianakis DN, Papaefthimiou M, Tsiveleka A and Spandidos DA: High prevalence of HPV-18 in correlation with ras gene mutations and clinicopathological parameters in cervical cancer studied from stained cytological smears. Oncol Rep 6: 1327-1331, 1999.

29. Aggelopoulou EP, Skarlos D, Papadimitriou C, Kittas C and Troungos C: Human papilloma virus DNA detection in oral lesions in the Greek population. Anticancer Res 19: 1391-1395, 1999.

30. Prokopakis P, Sourvinos G, Koumantaki Y, Koumantakis E and Spandidos DA: K-ras mutations and HPV infection in cervitis and intraepithelial neoplasias of the cervix. Oncol Rep 9: 129$133,2002$.

31. Roussaki-Schulze AV, Kouskoukis C, Rammos C, et al: Identification of human papillomavirus DNA in melanoma biopsy specimens of Greek population. Int J Clin Pharmacol Res 25: 145-150, 2005.

32. Lyronis ID, Baritaki S, Bizakis I, Tsardi M and Spandidos DA: Evaluation of the prevalence of human papillomavirus and Epstein-Barr virus in esophageal squamous cell carcinomas. Int J Biol Markers 20: 5-10, 2005

33. Mammas IN, Sourvinos G, Michael C and Spandidos DA: Human papilloma virus in hyperplastic tonsillar and adenoid tissues in children. Pediatr Infect Dis J 25: 1158-1162, 2006. 